\title{
A narrative review of current treatment strategies and emerging therapies in malignant pleural mesothelioma
}

\author{
Élise Di Lena ${ }^{1,2 \#}$, Ali Aboalsaud ${ }^{1 \#}$, Christian Sirois ${ }^{3}$, David Mulder ${ }^{3}$, Jonathan Spicer ${ }^{3}$, Lorenzo Ferri ${ }^{3}$, \\ Jonathan Cools-Lartigue ${ }^{3}$
}

${ }^{1}$ Department of Surgery, Division of General Surgery, McGill University Health Center, Montreal, Quebec, Canada; ${ }^{2}$ Department of Surgery, Division of Experimental Surgery, Goodman Cancer Research Center, McGill University, Montreal, Quebec, Canada; ${ }^{3}$ Department of Surgery, Division of Thoracic and Upper Gastrointestinal Surgery, Montreal General Hospital, Montreal, Quebec, Canada

Contributions: (I) Conception and Design: É Di Lena, A Aboalsaud, J Cools-Lartigue; (II) Administrative support: None; (III) Provision of study materials or patients: None; (IV) Collection and assembly of data: All authors; (V) Data analysis and interpretation: All authors; (VI) Manuscript writing: All authors; (VII) Final approval of manuscript: All authors.

\#These authors contributed equally to this work.

Correspondence to: Jonathan Cools-Lartigue. Division of Thoracic and Upper Gastrointestinal Surgery, Montreal General Hospital, 1650 Cedar Av., Montreal, Quebec, H3G 1A4, Canada. Email: jonathan.cools-lartigue@mcgill.ca.

\begin{abstract}
Malignant pleural mesothelioma is a rare but extremely lethal disease for which there is no single curative therapy. Given this, a significant lack of consensus exists regarding optimal treatment strategies. Patients typically present late in the course of their disease, which has led many to advocate for palliative approaches to therapy, while others believe that macroscopic resection of disease in conjunction with multimodal therapy is preferable in highly selected individuals. Surgical strategies have recently evolved from the maximally invasive extrapleural pneumonectomy (EPP) to the more conservative extended pleurectomy and decortication. The addition of chemotherapy and radiotherapy to surgical interventions have allowed to extend progression-free survival, but this is still measured in months rather than years. Moreover, there is a lack of high-quality evidence to support all interventions, leading to variations in guidelines regarding trimodal therapy and a paucity of data demonstrating superiority of a single approach to care. Given this, new and promising treatment options are emerging which may allow to better control and treat malignant pleural mesothelioma. These include immunotherapy, virotherapy, T-cell therapies, and vaccines, among others. This paper therefore aims to give a comprehensive overview of current available treatments with a focus on surgical and trimodal therapies, as well as emerging treatment options for malignant pleural mesothelioma.
\end{abstract}

Keywords: Malignant pleural mesothelioma (MPM); mesothelin; trimodal therapy

Received: 04 June 2020; Accepted: 13 August 2020; Published: 25 February 2021.

doi: $10.21037 /$ ccts-20-112

View this article at: http://dx.doi.org/10.21037/ccts-20-112

\section{Introduction}

Malignant mesothelioma is a disease which can occur in the pleura, the peritoneum, the pericardium, or the tunica vaginalis, though $90 \%$ of cases are malignant pleural mesothelioma (MPM) (1). Occupational asbestos exposure accounts for $85-90 \%$ of cases in men, and paraoccupational exposure accounts for the majority of cases in women, with a dose-dependent risk of MPM (1); other etiologies include radiation therapy, chronic pleural inflammation, chemical carcinogens, and possibly contamination of the polio vaccine prior to 1963 by simian virus-40 (2), as well as likely genetic factors $(3,4)$. The typical presentation of MPM includes males between 50 and 70 years of age presenting with either dyspnea secondary to an effusion or non-pleuritic chest wall pain from local invasion (3), with an insidious onset and chest $\mathrm{X}$-ray findings consistent with a pleural effusion in $80 \%$ to $95 \%$ of patients (2). The highest prevalence of cases is seen in the United Kingdom and the incidence is 
expected to peak in high-income countries roughly 40 years after asbestos regulations were implemented due to the long latency period (2), though they continue to rise and to be underreported in low- and middle-income countries (1). In the United States, it is thought that roughly $40 \%$ of the workforce was exposed between 1940 and 1979 (3). Despite this, MPM remains a rare and lethal disease, with only $2 \%$ to $10 \%$ of exposed individuals developing MPM and an expected survival of 6 months if left untreated (3). However, thanks to new diagnostic technologies including nextgeneration sequencing, the landscape of MPM is quickly changing, with new potential therapeutic targets being discovered constantly (5). This paper therefore aims to give a comprehensive overview of current available treatments as well as emerging therapies for MPM. We present the following article in accordance with the Narrative Review reporting checklist (available at https://ccts.amegroups.com/ article/view/10.21037/ccts-20-112/rc).

\section{Methods}

PubMed was searched using the keywords "malignant pleural mesothelioma" and "mesothelioma" from 2000 to April 2020. High-quality randomized-control trials, cohort and cross-sectional studies, and systematic and narrative reviews written in English or in French were evaluated for inclusion. North American and European society guidelines were also included and referenced. Manuscripts focusing on non-pleural malignant mesothelioma as well as case-reports were excluded.

\section{Current treatments}

\section{Surgery}

As early as 1922, and before MPM was found to be distinct from lung cancer, radical surgical intervention for diffuse MPM was advocated by Eiselsberg, who recommended pleurectomy for these cases, and radical pleuropneumonectomy or extrapleural pneumonectomy (EPP) (resection of pleura, lung, lymph nodes, ipsilateral pericardium and diaphragm) became one of the treatment options in the 1950s $(6,7)$. However, palliative treatments remained standard of care and few advocated for radical intervention given abysmal outcomes until Butchart et al. documented long-term cure in 2 of a 29 patient cohort, though in-hospital mortality rates were $31 \%$ and total complication rates were $44.8 \%$ (6).
Over time, as patient selection and perioperative care have improved, so have the surgical outcomes ( $8 \%$ perioperative mortality today compared to $33 \%$ in the 1970 s for EPP) (8), though survival is still measured in months rather than years and there has not been any significant improvement in survival over 4 decades $(9,10)$. Contrary to other oncologic resections, the aim of surgery for MPM is to perform maximal cytoreduction and to obtain a macroscopically complete (R1) resection and to use other local and systemic therapies as adjuncts, given the high morbidity and technical difficulty of aiming for an R0 resection (7,9,11-14). However, the role for surgical resection in MPM has been and remains controversial, with many societies advising against extensive surgical intervention outside of clinical trials $(15,16)$, while others are in favor of macroscopically complete surgical resection in patients with early-stage disease (11,17-20). Even among thoracic surgeons, there exist wide discrepancies in practice and beliefs regarding the curative potential of surgery for MPM (21). This variability in recommendations and practice is explained by various studies with conflicting outcomes, disease and treatment heterogeneity, and the lack of highquality randomized control trials (RCTs) (22). Review of data from the Surveillance, Epidemiology, and End Results (SEER) database demonstrated that cytoreductive surgery is associated with improved survival, leading to the authors' suggestion that surgically-centered therapy be the mainstay of treatment (10). Indeed, surgical intervention may confer a 9-month survival benefit, but its associated morbidity and mortality are high (23). Even in elderly patients, if properly selected, surgery may confer a survival benefit, though few are candidates for this option (24).

Among believers in surgery for MPM, there remains a lack of consensus regarding the optimal surgical intervention (25), and no society has taken a position in this regard $(9,26,27)$. There are strong advocates for EPP who believe that it offers the most oncologically complete resection, especially in earlier-stage cancers (please refer to Tables 1,2 for current staging) $(7,13,29)$. Another argument in favor of EPP is that resection of the lung allows for high-dose adjuvant radiation therapy (14). Retrospective data has shown some survival benefit from EPP in highly-selected patients, especially with neoadjuvant or adjuvant chemoradiotherapy (30), with a possible benefit in progression-free survival (PFS) (31). However, EPP is associated with significant morbidity $(25 \%)$ and mortality (4-15\%) (15). In fact, the 2011 Mesothelioma and Radical Surgery (MARS) trial comparing EPP to no EPP 
Table 1 Eight International Association for the Study of Lung Cancer (IASLC) TNM classification of malignant pleural mesothelioma (MPM) (28)

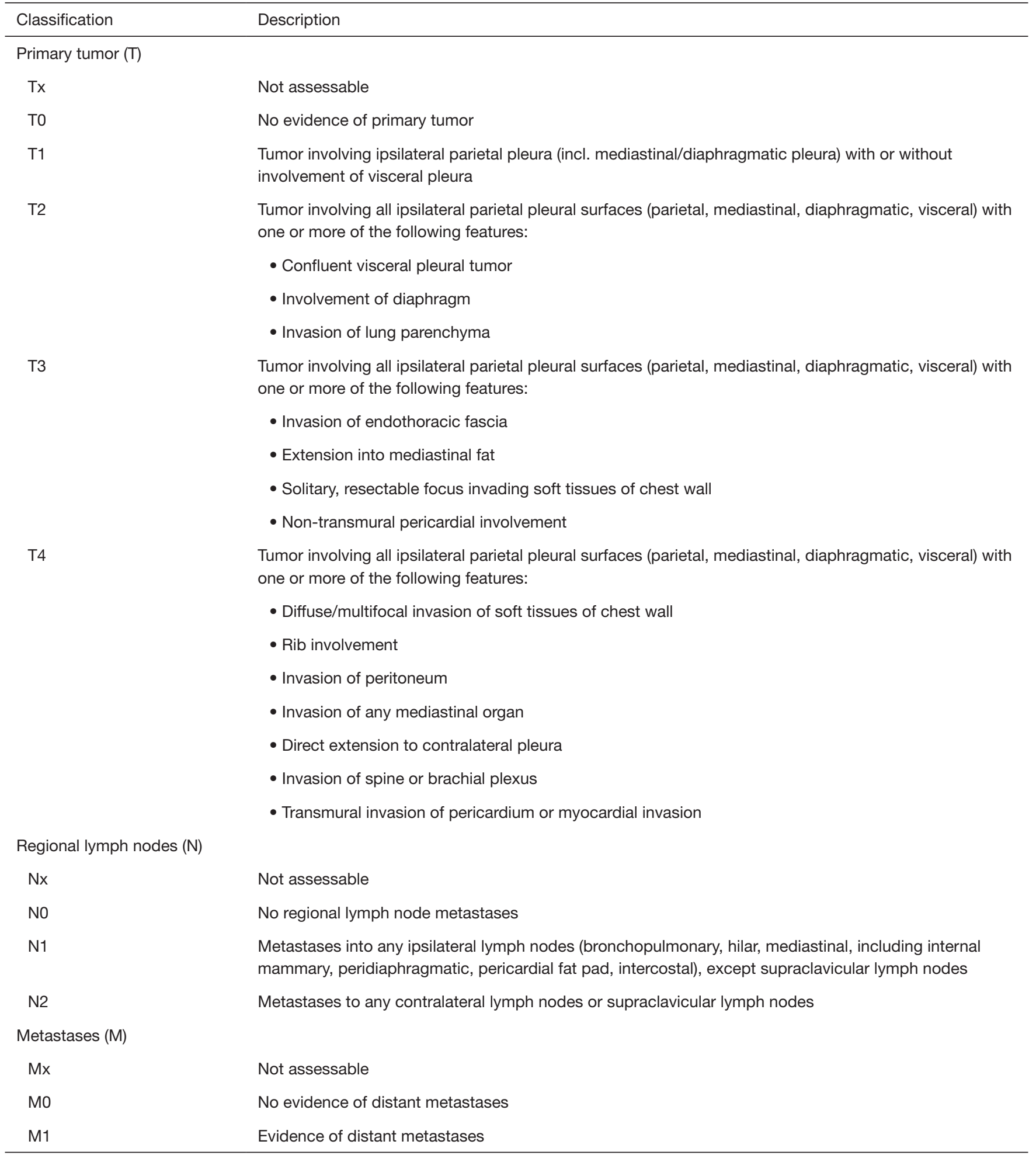


Table 2 Eight International Association for the Study of Lung Cancer (IASLC) TNM staging of malignant pleural mesothelioma (MPM) (28)

\begin{tabular}{lccc}
\hline Stage & T & N & M \\
\hline I IA & 1 & 0 & 0 \\
IB & 2,3 & 0 & 0 \\
II & 1,2 & 1 & 0 \\
III & & & \\
IIIA & 3 & 1 & 0 \\
IIIB & $1-3$ & 2 & 0 \\
IV & 4 & $0-2$ & 0 \\
& Any & Any & 1 \\
\hline
\end{tabular}

recommended against EPP given the high morbidity and 30-day mortality of the procedure, though this trial was a feasibility study and was underpowered (32). Considering this, other experts advocate for the less morbid pleurectomy and decortication (PD) (also referred to as extended PD), during which the visceral and parietal pleurae are resected, and occasionally the pericardium and diaphragm, but not the lung $(7,13)$. Some studies have found comparable survival outcomes with significantly less morbidity and mortality associated with this procedure $(8,31,33,34)$. Furthermore, certain systematic reviews have found a survival benefit in favor of $\mathrm{PD}$, likely secondary to the decreased peri-operative mortality $(35,36)$, and PD may also offer improved quality of life $(34,37)$, leading many to favor PD today in the treatment of MPM. However, other retrospective studies, systematic reviews and propensity matched comparisons have noted no difference in morbidity and mortality between the two procedures (38-40). The data is therefore extremely heterogeneous (12). Overall, most experts agree that patients should be evaluated as part of a multi-modal team and proper RCTs must be conducted to further determine the best surgical intervention in MPM $(12,22,26,41-43)$. The MARS-2 trial is currently underway, looking at whether PD with neoadjuvant chemotherapy offers a survival benefit compared to chemotherapy alone, and preliminary results should be made available in September $2020(26,44,45)$.

\section{Trimodal therapy}

Few RCTs have been conducted comparing multimodality therapy to use of chemotherapy or surgical resection alone, and the recommendations for chemotherapy as part of trimodal therapy have mostly been extrapolated from these few trials, feasibility trials, and trials evaluating effects of chemotherapy in unresectable disease. Several societies recommend the use of trimodal therapy (surgery, chemotherapy, radiation therapy) $(11,20,46,47)$, though the British Thoracic Society and the European Respiratory Society suggest that multimodal therapy only be used in the context of a clinical trial $(15,48)$; however the appropriate timing of therapy remains unknown, with no clear evidence for the sequence in which to provide various treatments (48). Furthermore, even with trimodal therapy, long-term survival is poor, with $10 \%$ of patients surviving at 5 years (49).

General recommendations for chemotherapy are a platinum-based therapy [cisplatin, typically, or carboplatin in older or more frail patients $(50,51)]$ in addition to pemetrexed (folate antimetabolite) with B12 and folic acid supplementation $(11,15,20,46-48)$. The recommendations for the addition of pemetrexed emerged from RCTs which demonstrated improved survival (by 2.8 months) compared to cisplatin alone, with B12 and folic acid reducing toxicity $(52,53)$. In patients with a poor functional status or in elderly patients, carboplatin offers adequate survival benefits and is recommended in combination with pemetrexed (54). Bevacizumab (vascular endothelial growth factor (VEGF) inhibitor) may also be added to standard chemotherapy in patients with a good performance status $(11,15,20)$. This recommendation is derived from a large phase III trial, MAPS, comparing pemetrexed/ cisplatin alone to this combination with bevacizumab (55). This study demonstrated improved overall survival (by 2.7 months) with the addition of the VEGF inhibitor; however, this study was only performed in unresectable MPM (55). No second-line chemotherapy is generally specifically recommended, and it is advised that patients be enrolled in a clinical trial or offered palliative care $(11,15,46,48)$. The American Society of Clinical Oncology (ASCO) and the National Comprehensive Cancer Network (NCCN) suggest possible use of vinorelbine as secondline therapy in patients who cannot be enrolled in a trial $(11,20)$, with NCCN also suggesting immunotherapy and rechallenge with chemotherapy as second-line (20). Second-line pemetrexed has shown some effect on tumor response and delayed disease progression when compared to best supportive care in a phase III trial (56). The recommendation for vinorelbine stems from an RCT demonstrating a trend in improved survival (non-statistically 
significant) in patients treated with vinorelbine compared to active symptom control (57). Other treatments being investigated include PD with intraoperative intracavitary hyperthermic chemotherapy, though no society recommends this treatment outside of clinical trials; more evidence is required before this treatment option can be recommended (58-66).

Most studies evaluating trimodal therapy have either been retrospective reviews or feasibility studies with no control or randomization, and have used historical data as a comparator (67-73). In 2007, a multicenter trial was published evaluating the feasibility of neoadjuvant chemotherapy (3 cycles of cisplatin/gemcitabine) followed by EPP in all patients and the recommendation of adjuvant radiation (74). Operability was $74 \%$, with resectability of $61 \%$, with an intention-to-treat median overall survival of 19.8 months compared to 23 months in those who underwent EPP (74). A similar study was published the same year by an Italian group with similar results (75). In 2009, a multicenter phase II trial was published evaluating neoadjuvant cisplatin/pemetrexed in the context of trimodal therapy (76). Patients with stage I-III MPM received 4 cycles of cisplatin/pemetrexed and then those without disease progression underwent EPP followed by adjuvant hemithoracic radiation (76). Patients who completed all therapies had a median survival of 29.1 months with a 2 -year survival of $61.2 \%$, and the primary endpoint of pathological complete response was achieved in $5 \%$ of patients; however, by intention-to-treat analysis, the median survival was only 16.8 months with a 2-year survival of $37.2 \%$ (76). This study led to the authors' conclusion that, in highly selected patients, trimodal therapy may be beneficial (76). A similar multicenter phase II study was published in 2010 by the European Organisation for Research and Treatment of Cancer (EORTC) with the primary end-point defined as "success of treatment" (77). Seventy-four percent of patients underwent EPP and $65 \%$ completed adjuvant radiotherapy, but only $42 \%$ of patients met the definition of success, leading the authors to conclude that, "although feasible, trimodality therapy in patients with mesothelioma was not completed within the strictly defined timelines of this protocol and adjustments are necessary" (77). Overall, though feasible in certain patients, trimodal therapy involving neoadjuvant chemotherapy, EPP, and adjuvant radiotherapy proves to be challenging for patients, with low completion rates, and poor results in patients with $\mathrm{N} 2$ disease (contralateral lymph node metastases, see Table 1) or biphasic/sarcomatoid histology $(70,74)$.
As surgical practice has shifted from EPP to PD, few studies evaluating trimodal therapy with $\mathrm{PD}$ have been published. In 2012, a study was published attempting to compare EPP to PD in the trimodal setting (8). This was a non-randomized, prospective trial comparing neoadjuvant chemotherapy (3 cycles of either cisplatin/gemcitabine or cisplatin/pemetrexed) followed by EPP and adjuvant radiotherapy to $\mathrm{PD}$ with hyperthermic pleural lavage with povidone-iodine and adjuvant chemotherapy (4-6 cycles of either cisplatin/gemcitabine or cisplatin/pemetrexed) and prophylactic radiotherapy (8). Sixty-eight percent of patients completed all treatment in the EPP group, with 2 patients surviving more than 50 months (median survival 12.8 months) (8). A majority of patients $(96.3 \%)$ completed all treatment in the PD group, with a median survival of 23 months in the PD group compared to 12.8 months in the EPP group, leading the authors to conclude that PD was more feasible and had better outcomes than EPP in the context of trimodal therapy (8).

Specifically regarding the addition of adjuvant or neoadjuvant radiation therapy, the data is similarly mixed, uncontrolled and nonrandomized. Classically, hemithoracic radiation involved irradiating the entire pleural cavity, including ipsilateral nodal beds, with photon/electron radiation using anteroposterior/posteroanterior fields and blocks shielding vital organs, in the context of EPP where lung-associated complications are not a concern (78). However, it remained difficult to spare other vital organs (esophagus, heart, bowel, etc.) from radiation exposure as well as challenging to target the posterior diaphragmatic sulcus (79). Intensity-modulated radiation therapy (IMRT) was initially developed for use in head-and-neck and prostate cancers, using three-dimensional computerized planning to allow radiation doses to conform more closely with target areas $(80,81)$. In 2007 , a prospective study evaluating local control with adjuvant IMRT following EPP (with or without chemotherapy) demonstrated improved median and 3 -year survival, with only $5 \%$ of irradiated patients having a local recurrence within the irradiated field (79). In 2013, a retrospective study also demonstrated safety and improved local recurrence rates with adjuvant IMRT following EPP (82), and feasibility was further demonstrated by another phase II trial (83). In 2014, a European group attempted a multicenter, international RCT (SAKK 17/04) to evaluate the effect of the addition of adjuvant IMRT compared to neoadjuvant cisplatin/ pemetrexed and EPP alone (84). Unfortunately, accrual was slow and the primary endpoint of 1-year relapse- 
free survival could not be reached, leading the authors to advocate against the use of routine hemithoracic adjuvant radiotherapy (84). The 2014 SMART trial aimed to evaluate neoadjuvant IMRT in T1-3N0 MPM followed by EPP with adjuvant chemotherapy (cisplatin and an anti-folate) offered to those with ypN2 disease [ipsilateral or subcarinal nodal involvement, using the $7^{\text {th }}$ edition of the International Association for the Study of Lung Cancer (IASLC) TNM classification] $(5,85)$. The 3 -year survival was $84 \%$ in the epithelial subtype, compared to $13 \%$ in the biphasic subtype; however, there was no comparison to adjuvant radiotherapy (85). Another study, published the same year, evaluated adjuvant radiotherapy following $\mathrm{PD}$, with most patients $(95 \%)$ receiving either adjuvant, neoadjuvant, or sandwich chemotherapy (86). This study demonstrated a median overall survival of 33 months and PFS of 29 months (86). Given the need for close monitoring and adequate selection of patients, this approach is not generally widely recommended outside of highly specialized centers (78). In 2016, a group from Memorial Sloan Kettering Cancer Center (MSKCC) published results of the IMPRINT phase II trial, demonstrating feasibility and acceptable complication rates associated with adjuvant IMRT following neoadjuvant chemotherapy and PD (87); the following year, the same group retrospectively compared PD trimodal therapy with conventional radiotherapy to hemithoracic IMRT, finding improved overall survival in those who had received IMRT (20.2 months compared to 12.3 months) (88). Overall, completion of adjuvant IMRT remains low at roughly two-thirds, regardless of whether patients receive EPP or PD (89).

At least in the United States, where both ASCO and NCCN guidelines recommend multimodality treatment, this seems to be underutilized, with up to $29 \%$ of patients with stage I-III epithelioid MPM not receiving curative-intent therapy, especially in low-volume settings (90). A review of the National Cancer Database further demonstrated that only $20 \%$ of patients receive cancer-directed surgery, $2.6 \%$ of whom receive trimodal therapy (91), which may be a result of the lack of compelling data and demonstrate a need for further well-conducted RCTs (44). Systematic reviews have shown the failure of enrolment in RCTs, the need for further phase II trials including a control group, and the need to systematically publish intention-to-treat analyses $(92,93)$. The upcoming MARS-2 results in September 2020 (see section 'Surgery') may help clarify the benefit of multimodality treatment (45). Additionally, the MSKCC group should be publishing results of their phase II toxicity study comparing adjuvant versus neoadjuvant platinum/pemetrexed in combination with PD and adjuvant IMRT in July 2021; this may help clarify the optimal order of chemotherapy in trimodal therapy (94).

\section{Emerging therapies and future prospects}

Current treatment of malignant mesothelioma includes surgery, chemotherapy, and radiation therapy. However, prognosis remains poor despite multimodality treatment with overall survival being 9 to 17 months after diagnosis $(75,95,96)$. In light of this, a number of new emerging and experimental treatments are becoming available for patients not responding to conventional treatment, including immunotherapy, T-cell therapies, anti-tumor vaccines, and virotherapy.

\section{Immunotherapy}

The immune system plays an important role in MPM with interactions between the tumor and the immune system being driven mostly by local immunoregulatory mechanisms, and there is evidence of systemic response to tumor-directed immunotherapies $(97,98)$. Patients with MPM have shown improved survival when tumors were highly infiltrated by cytotoxic CD8+ T cells (tumor-infiltrating lymphocytes), whereas programmed death-ligand 1 (PD-L1) expression is associated with reduced survival (median OS 5 months in patients who are PD-L1-positive vs. 14.5 months PD-L1negative patients; $\mathrm{P}<0.0001)(99-101)$. Off-label usage of anti-PD-1 antibodies pembrolizumab and nivolumab as single agents, or nivolumab with the cytotoxic $\mathrm{T}$ lymphocyte antigen 4 (CTLA-4) antibody ipilimumab have shown promising activity $(102,103)$.

Popat et al. presented preliminary results of a phase III trial in which 144 patients with advanced pre-treated MPM were randomised to either pembrolizumab or standard chemotherapy. Pembrolizumab improved the objective response rate $(22 \%)$ relative to gemcitabine or vinorelbine (6\%). However, PFS (2.5 vs. 3.4 months) as well as overall survival (10.7 vs. 11.7 months) were similar in both groups (102). In another trial, Alley et al. treated 25 patients with PD-L1-positive MPM with pembrolizumab. Five (20\%) patients had a partial response and $13(52 \%)$ had stable disease (104).

Other immunotherapies have been assessed. For example, a multicenter randomised, non-comparative, open-label, phase 2 trial (MAPS2) conducted in 21 hospitals in France 
aiming to assess nivolumab alone or in combination with ipilimumab achieved 12-week disease control in 24 (44\%) out of 54 patients who received nivolumab, and in 27 (50\%) out of 54 receiving nivolumab plus ipilimumab. Objective responses were 10 (19\%) with nivolumab and 15 (28\%) with nivolumab plus ipilimumab (103). Another singlearm, phase II trial (INITIATE) assessed the combination of ipilimumab and nivolumab for the treatment of recurrent MPM. In the study, 34 patients were evaluated for response at 12 weeks. Ten patients (29\%) had a partial response and 13 patients $(38 \%)$ had stable disease. However, adverse events were reported in 33 patients (94\%) with 12 patients (34\%) reporting grade 3 toxicity (105). These findings show promising activity of both single and double agent blockade in MPM.

Another CTLA-4 inhibitor, tremelimumab, was studied in a randomized phase II trial (DETERMINE) in 571 patients but did not provide survival benefit compared to placebo (median 7.7 vs. 7.3 months, respectively) (106). When combined with the anti-PD-L1 antibody durvalumab in a phase II study, there was suggestion of activity as 11 (28\%) out of 40 patients had an immune-related objective response (all partial responses) with a median response duration of 16.1 months, and 25 patients $(63 \%)$ had disease control with median PFS of 5.7 months and median overall survival of 16.6 month (107). However, randomized data are required.

Older immunotherapeutic approaches using interferons or interleukin-2, either alone or in combination with chemotherapy, have not offered substantive advantage (108-110).

\section{T-cell therapies}

Mesothelin (MSLN) is a recently characterised cell-surface glycoprotein and biomarker which is expressed on normal mesothelial cells (111), but also on malignant mesothelioma and other solid tumors' cell surface (5). It has been found to shed from malignant mesothelioma cells and certain other solid tumors (112), and serum levels of MSLN may be elevated in as many as $80-84 \%$ of patients with MPM $(113,114)$. Adoptive T-cell therapy represents a promising new strategy in MPM. A phase I trial investigating chimeric antigen receptor (CAR) T-cell therapy targeting MSLN in MPM patients recently reported encouraging results. In the study, 18 patients were treated with a single dose of CD28costimulated MSLN CAR-T cells with the I-caspase-9 safety gene administered intrapleurally with or without cyclophosphamide preconditioning. Fourteen patients received subsequent anti-PD-1 therapy, off-protocol. Of those 14 patients, 2 had complete metabolic response on PET, 5 had partial responses, and 4 had stable disease. Regarding safety, no CAR-T cell-related toxicities higher than grade 1 were observed (115). This shows promise for future developments especially when combining CAR-T cell therapy with anti-PD-1 therapy giving previous preclinical data showing that CAR-T cells become functionally exhausted in the presence of a large tumor burden and, in some patients, CAR-T cells expand following PD-1 blockade (116).

\section{Vaccines}

Another way to prime acquired anti-tumoral activity of the immune system is vaccination, which has led to significant research on vaccine therapy in MPM. One promising candidate is the Wilms tumor-1 (WT1) protein in MPM which is highly expressed compared to normal (117), making it an ideal target for a tumor-selective cancer vaccine. A double-blind, controlled, two center phase II trial randomized 41 pre-treated MPM patients to either galinpepimut-S, a WT1 analogue peptide vaccine, with Granulocyte-macrophage colony-stimulating factor (GMCSF) and Montanide, which are immunologic adjuvants, or GM-CSF and Montanide alone. The vaccine arm had improved PFS at 1 year (45\%) compared to the control arm (33\%) with median PFS of 10.1 vs. 7.4 months. Median overall survival was 22.8 months in the vaccine group versus 18.3 months in the control group. However, this study was not powered for comparison between the treatment arms (118). This shows promise for new treatment modalities given the limited treatment options for MPM.

Another therapeutic target is dendritic cell (DC) therapy which is a cell-based vaccination used to instigate an antitumor immune response. DCs present tumor-associated antigens (TAAs) to the T-cells in lymph nodes, inducing proliferation and activation of tumor-specific CD4+ and CD8+ T-cells. DC function is impaired in cancer patients $(119,120)$. In DC therapy, DCs are matured and activated $e x$ vivo to enhance their immunogenic function and circumvent tumor immunosuppression, and they are then loaded with TAAs. Phase I trials demonstrated promising clinical response with long-lasting radiological response $(121,122)$. Recently, a multicenter, randomized phase II/III trial was initiated with the aim of assessing DCs loaded with allogenic tumor cell lysate (MesoPher) in MPM patients after chemotherapy 
(DENIM trial) to evaluate whether this will improve survival and prove effective as a maintenance therapy (123).

\section{Virotberapy}

Oncolytic virotherapy has recently emerged as a promising experimental modality. Virus vectors are used to infect tumor cells, leading to cell lysis while releasing tumorassociated and viral antigens and thus triggering an antitumor immune response $(124,125)$. In most viral platforms, the preferred route of administration is intratumoral (IT) which makes MPM an ideal model for the study of oncolytic virotherapy given the accessibility of the pleural cavity and the pattern of growth $(126,127)$. Adenovirus has been the most studied oncolytic virus preclinical and clinical trials. It has shown good results in animal studies with tumor regression and improved survival (128). Human trials using adenoviral vectors have shown good safety profiles, but low response rates (129-131).

Herpes simplex virus type $1(\mathrm{HSV}-1)$ is another virus that has been studied in preclinical trials, but no human trials have been published. An ongoing phase I/IIa trial is seeking to evaluate the safety and biological effects of single and multiple administrations of HSV1716, an oncolytic virus which is a mutant HSV-1, in the treatment of MPM (132).

\section{Other investigational therapies}

Other systemic treatment modalities have been studied, but none so far are indicated outside the setting of a clinical trial. For example, nintedanib [a vascular endothelial growth factor receptor (VEGFR), platelet-derived growth factor receptor (PDGFR), and fibroblast growth factor receptor (FGFR) tyrosine-kinase inhibitor] has shown promising benefits in PFS in a phase II trial when added to conventional chemotherapy (133), but those benefit were not reproducible once a phase III trial was conducted (134). Similarly, Vorinostat, an oral histone deacetylase inhibitor, showed promise in an early trial (135), but when a phase III trial involving 661 patients was conducted, there was no significant benefit compared to placebo (136).

\section{Conclusions}

MPM is a rare tumor predominantly caused by asbestos exposure with a very prolonged latency period. It remains lethal with poor outcomes despite aggressive therapy as patients typically present with advanced disease. Given this, surgical intervention remains controversial, and is typically reserved for patients with earlier-stage disease who are candidates for multimodal therapy. Surgical approach is also contentious, though recently surgical culture has shifted from the historically preferred but highly morbid EPP to the more conservative extended PD, while aiming for an $\mathrm{R} 1$ resection. Additional chemoradiotherapy is also the mainstay of treatment, though there is a lack of evidence regarding the optimal timing of such interventions. Overall, patient selection remains critical, especially given the lack of consensus.

Other treatment options are beginning to emerge and continue to evolve as our understanding of the disease improves. In the last few years, the genetics, immunebiology, biomarkers, and tumor microenvironment have been studied, and the knowledge derived has thus opened the door to many emerging therapies. Currently, there are multiple clinical trials interrogating various treatment modalities as well as combination therapies. However, evidence supporting the use of these new therapeutic modalities remains scarce mainly due to the lack of randomized trials.

Given the rarity of the disease and the lack of success with a one-size-fits-all approach in MPM patients, scientific collaborations are warranted to conduct well designed studies that will attempt to slow disease progression, decrease morbidity of current therapies, and improve patient survival.

\section{Acknowledgments}

Funding: None.

\section{Footnote}

Reporting Checklist: The authors have completed the Narrative Review reporting checklist. Available at https:// ccts.amegroups.com/article/view/10.21037/ccts-20-112/rc

Conflicts of Interest: All authors have completed the ICMJE uniform disclosure form (available at https://ccts. amegroups.com/article/view/10.21037/ccts-20-112/coif). The authors have no conflicts of interest to declare.

Ethical Statement: The authors are accountable for all aspects of the work in ensuring that questions related to the accuracy or integrity of any part of the work are appropriately investigated and resolved. 
Open Access Statement: This is an Open Access article distributed in accordance with the Creative Commons Attribution-NonCommercial-NoDerivs 4.0 International License (CC BY-NC-ND 4.0), which permits the noncommercial replication and distribution of the article with the strict proviso that no changes or edits are made and the original work is properly cited (including links to both the formal publication through the relevant DOI and the license). See: https://creativecommons.org/licenses/by-nc-nd/4.0/.

\section{References}

1. Lucchini RG, Hashim D, Lambertini L, et al. Evidence From Epidemiology and Health Surveillance. Malignant Pleural Mesothelioma: Elsevier; 2019: 1-13.

2. Cugell DW, Kamp DW. Asbestos and the pleura: a review. Chest 2004;125:1103-17.

3. Ismail-Khan R, Robinson LA, Williams CC Jr, et al. Malignant pleural mesothelioma: a comprehensive review. Cancer Control 2006;13:255-63.

4. Hassan R, Morrow B, Thomas A, et al. Inherited predisposition to malignant mesothelioma and overall survival following platinum chemotherapy. Proc Natl Acad Sci U S A 2019;116:9008-13.

5. Mutti L, Peikert T, Robinson BWS, et al. Scientific Advances and New Frontiers in Mesothelioma Therapeutics. J Thorac Oncol 2018;13:1269-83.

6. Butchart EG, Ashcroft T, Barnsley W, et al. Pleuropneumonectomy in the management of diffuse malignant mesothelioma of the pleura. Experience with 29 patients. Thorax 1976;31:15-24.

7. Sugarbaker DJ, Wolf AS. Surgery for malignant pleural mesothelioma. Expert Rev Respir Med 2010;4:363-72.

8. Lang-Lazdunski L, Bille A, Lal R, et al. Pleurectomy/ decortication is superior to extrapleural pneumonectomy in the multimodality management of patients with malignant pleural mesothelioma. J Thorac Oncol 2012;7:737-43.

9. Chauhan D, Vigneswaran WT. History of Pleural Surgical Treatment. In: Hesdorffer M, Bates-Pappas GE, editors. Caring for Patients with Mesothelioma: Principles and Guidelines. Cham: Springer International Publishing; 2019: 3-11.

10. Taioli E, Wolf AS, Camacho-Rivera M, et al. Determinants of Survival in Malignant Pleural Mesothelioma: A Surveillance, Epidemiology, and End Results (SEER) Study of 14,228 Patients. PLoS One 2015;10:e0145039.

11. Kindler HL, Ismaila N, Armato SG 3rd, et al. Treatment of Malignant Pleural Mesothelioma: American Society of Clinical Oncology Clinical Practice Guideline. J Clin Oncol 2018;36:1343-73.

12. Bueno R, Opitz I, Taskforce IM. Surgery in Malignant Pleural Mesothelioma. J Thorac Oncol 2018;13:1638-54.

13. Flores RM. Pleurectomy decortication for mesothelioma: The procedure of choice when possible. J Thorac Cardiovasc Surg 2016;151:310-2.

14. Sugarbaker DJ. Macroscopic complete resection: the goal of primary surgery in multimodality therapy for pleural mesothelioma. J Thorac Oncol 2006;1:175-6.

15. Woolhouse I, Bishop L, Darlison L, et al. BTS guideline for the investigation and management of malignant pleural mesothelioma. BMJ Open Respir Res 2018;5:e000266.

16. Stahel RA, Weder W, Lievens Y, et al. Malignant pleural mesothelioma: ESMO Clinical Practice Guidelines for diagnosis, treatment and follow-up. Ann Oncol 2010;21:v126-8.

17. Rusch V, Baldini EH, Bueno R, et al. The role of surgical cytoreduction in the treatment of malignant pleural mesothelioma: meeting summary of the International Mesothelioma Interest Group Congress, September 11-14, 2012, Boston, Mass. J Thorac Cardiovasc Surg 2013;145:909-10.

18. Klikovits T, Hoda MA, Dong Y, et al. Management of malignant pleural mesothelioma - part 3 : Data from the Austrian Mesothelioma Interest Group (AMIG) database. Wien Klin Wochenschr 2016;128:627-34.

19. Pinto C, Novello S, Torri V, et al. Second Italian Consensus Conference on Malignant Pleural Mesothelioma: State of the art and recommendations. Cancer Treat Rev 2013;39:328-39.

20. NCCN. NCCN Clinical Practice Guidelines in Oncology - Malignang Pleural Mesothelioma: National Comprehensive Cancer Network; 2019 [updated November 27, 2019]. Available online: https://www.nccn. org/professionals/physician_gls/pdf/mpm.pdf

21. Treasure T, Fiorentino F, Utley M, et al. A survey of opinions and beliefs concerning surgery for malignant pleural mesothelioma amongst 802 members of the european association for cardio-thoracic surgery (EACTS), the European society of thoracic surgeons (ESTS) and the society of thoracic surgeons (STS). Interact Cardiovasc Thorac Surg 2011;12:341-6.

22. Treasure T. What is the best approach for surgery of malignant pleural mesothelioma? It is to put our efforts into obtaining trustworthy evidence for practice. J Thorac Cardiovasc Surg 2016;151:307-9.

23. Utley M, Fiorentino F, Treasure T. Obtaining an upper 
estimate of the survival benefit associated with surgery for mesothelioma. Eur J Cardiothorac Surg 2010;38:241-4.

24. Verma V, Wegner RE, Ludmir EB, et al. Management of Malignant Pleural Mesothelioma in the Elderly Population. Ann Surg Oncol 2019;26:2357-66.

25. Takuwa T, Hasegawa S. Current surgical strategies for malignant pleural mesothelioma. Surg Today 2016;46:887-94.

26. Datta A, Smith R, Fiorentino F, et al. Surgery in the treatment of malignant pleural mesothelioma: recruitment into trials should be the default position. Thorax 2014;69:194-7.

27. Hasegawa S. Extrapleural pneumonectomy or pleurectomy/ decortication for malignant pleural mesothelioma. Gen Thorac Cardiovasc Surg 2014;62:516-21.

28. Berzenji L, Van Schil PE, Carp L. The eighth TNM classification for malignant pleural mesothelioma. Transl Lung Cancer Res 2018;7:543-9.

29. Rusch VW, Giroux D, Kennedy C, et al. Initial Analysis of the International Association For the Study of Lung Cancer Mesothelioma Database. J Thorac Oncol 2012;7:1631-9.

30. Spaggiari L, Marulli G, Bovolato P, et al. Extrapleural pneumonectomy for malignant mesothelioma: an Italian multicenter retrospective study. Ann Thorac Surg 2014;97:1859-65.

31. Flores RM, Pass HI, Seshan VE, et al. Extrapleural pneumonectomy versus pleurectomy/decortication in the surgical management of malignant pleural mesothelioma: results in 663 patients. J Thorac Cardiovasc Surg 2008;135:620-6.

32. Treasure T, Lang-Lazdunski L, Waller D, et al. Extra-pleural pneumonectomy versus no extra-pleural pneumonectomy for patients with malignant pleural mesothelioma: clinical outcomes of the Mesothelioma and Radical Surgery (MARS) randomised feasibility study. Lancet Oncol 2011;12:763-72.

33. Batirel HF, Metintas M, Caglar HB, et al. Adoption of pleurectomy and decortication for malignant mesothelioma leads to similar survival as extrapleural pneumonectomy. J Thorac Cardiovasc Surg 2016;151:478-84.

34. Rena O, Casadio C. Extrapleural pneumonectomy for early stage malignant pleural mesothelioma: a harmful procedure. Lung Cancer 2012;77:151-5.

35. Cao C, Tian D, Park J, et al. A systematic review and meta-analysis of surgical treatments for malignant pleural mesothelioma. Lung Cancer 2014;83:240-5.

36. Magouliotis DE, Tasiopoulou VS, Athanassiadi K. Updated meta-analysis of survival after extrapleural pneumonectomy versus pleurectomy/decortication in mesothelioma. Gen Thorac Cardiovasc Surg 2019;67:312-20.

37. Vigneswaran WT, Kircheva DY, Rodrigues AE, et al. Influence of Pleurectomy and Decortication in HealthRelated Quality of Life Among Patients with Malignant Pleural Mesothelioma. World J Surg 2018;42:1036-45.

38. Papaspyros S, Papaspyros S. Surgical Management of Malignant Pleural Mesothelioma: Impact of Surgery on Survival and Quality of Life-Relation to Chemotherapy, Radiotherapy, and Alternative Therapies. ISRN Surg 2014;2014:817203.

39. Kostron A, Friess M, Inci I, et al. Propensity matched comparison of extrapleural pneumonectomy and pleurectomy/decortication for mesothelioma patients. Interact Cardiovasc Thorac Surg 2017;24:740-6.

40. Sharkey AJ, Tenconi S, Nakas A, et al. The effects of an intentional transition from extrapleural pneumonectomy to extended pleurectomy/decortication. Eur J Cardiothorac Surg 2016;49:1632-41.

41. Treasure T, Sedrakyan A. Pleural mesothelioma: little evidence, still time to do trials. Lancet 2004;364:1183-5.

42. Fournel L, Janet-Vendroux A, Canny-Hamelin E, et al. Malignant pleural mesothelioma: The role of surgery. Rev Pneumol Clin 2018;74:351-8.

43. Treasure T, Macbeth F. Fifteen years in the evaluation of extrapleural pneumonectomy: Lessons to be learned. J Thorac Cardiovasc Surg 2015;149:1382-3.

44. Hiddinga BI, van Meerbeeck JP. Surgery in mesothelioma--where do we go after MARS? J Thorac Oncol 2013;8:525-9.

45. ClinicalTrials.gov. Mesothelioma and Radical Surgery 2 (MARS2) 2019 [updated October 20, 2019]. Available online: https://clinicaltrials.gov/ct2/show/NCT02040272

46. Baas P, Fennell D, Kerr KM, et al. Malignant pleural mesothelioma: ESMO Clinical Practice Guidelines for diagnosis, treatment and follow-up. Ann Oncol 2015;26:v31.

47. van Zandwijk N, Clarke C, Henderson D, et al. Guidelines for the diagnosis and treatment of malignant pleural mesothelioma. J Thorac Dis 2013;5:E254.

48. Scherpereel A, Astoul P, Baas P, et al. Guidelines of the European Respiratory Society and the European Society of Thoracic Surgeons for the management of malignant pleural mesothelioma. Eur Respir J 2010;35:479-95.

49. Fahrner R, Ochsenbein A, Schmid RA, et al. Long term survival after trimodal therapy in malignant pleural mesothelioma. Swiss Med Wkly 2012;142:w13686. 
50. Ceresoli GL, Castagneto B, Zucali PA, et al. Pemetrexed plus carboplatin in elderly patients with malignant pleural mesothelioma: combined analysis of two phase II trials. Br J Cancer 2008;99:51-6.

51. Katirtzoglou N, Gkiozos I, Makrilia N, et al. Carboplatin Plus Pemetrexed as First-line Treatment of Patients With Malignant Pleural Mesothelioma: A Phase II Study. Clin Lung Cancer 2010;11:30-5.

52. Vogelzang NJ, Rusthoven JJ, Symanowski J, et al. Phase III Study of Pemetrexed in Combination With Cisplatin Versus Cisplatin Alone in Patients With Malignant Pleural Mesothelioma. J Clin Oncol 2003;21:2636-44.

53. Scagliotti GV, Shin DM, Kindler HL, et al. Phase II study of pemetrexed with and without folic acid and vitamin B12 as front-line therapy in malignant pleural mesothelioma. J Clin Oncol 2003;21:1556-61.

54. Ceresoli GL, Zucali PA, Favaretto AG, et al. Phase II Study of Pemetrexed Plus Carboplatin in Malignant Pleural Mesothelioma. J Clin Oncol 2006;24:1443-8.

55. Zalcman G, Mazieres J, Margery J, et al. Bevacizumab for newly diagnosed pleural mesothelioma in the Mesothelioma Avastin Cisplatin Pemetrexed Study (MAPS): a randomised, controlled, open-label, phase 3 trial. Lancet 2016;387:1405-14.

56. Jassem J, Ramlau R, Santoro A, et al. Phase III trial of pemetrexed plus best supportive care compared with best supportive care in previously treated patients with advanced malignant pleural mesothelioma. J Clin Oncol 2008;26:1698-704.

57. Muers MF, Stephens RJ, Fisher P, et al. Active symptom control with or without chemotherapy in the treatment of patients with malignant pleural mesothelioma (MS01): a multicentre randomised trial. Lancet 2008;371:1685-94.

58. Richards WG, Zellos L, Bueno R, et al. Phase I to II study of pleurectomy/decortication and intraoperative intracavitary hyperthermic cisplatin lavage for mesothelioma. J Clin Oncol 2006;24:1561-7.

59. Gomez D, Tsao AS. Local and systemic therapies for malignant pleural mesothelioma. Curr Treat Options Oncol 2014;15:683-99.

60. Burt BM, Richards WG, Lee HS, et al. A Phase I Trial of Surgical Resection and Intraoperative Hyperthermic Cisplatin and Gemcitabine for Pleural Mesothelioma. J Thorac Oncol 2018;13:1400-9.

61. Bertoglio P, Aprile V, Ambrogi MC, et al. The role of intracavitary therapies in the treatment of malignant pleural mesothelioma. J Thorac Dis 2018;10:S293-7.

62. Opitz I, Lauk O, Meerang M, et al. Intracavitary cisplatin- fibrin chemotherapy after surgery for malignant pleural mesothelioma: A phase I trial. J Thorac Cardiovasc Surg 2019. [Epub ahead of print]. doi: 10.1016/ j.jtcvs.2019.07.073.

63. Okabe K. Intraoperative intracavitary hyperthermic chemotherapy for malignant pleural mesothelioma. Ann Transl Med 2017;5:233.

64. Ried M, Potzger T, Braune N, et al. Local and systemic exposure of cisplatin during hyperthermic intrathoracic chemotherapy perfusion after pleurectomy and decortication for treatment of pleural malignancies. J Surg Oncol 2013;107:735-40.

65. Ishibashi H, Kobayashi M, Takasaki C, et al. Interim results of pleurectomy/decortication and intraoperative intrapleural hyperthermic cisplatin perfusion for patients with malignant pleural mesothelioma intolerable to extrapleural pneumonectomy. Gen Thorac Cardiovasc Surg 2015;63:395-400.

66. Sugarbaker DJ, Gill RR, Yeap BY, et al. Hyperthermic intraoperative pleural cisplatin chemotherapy extends interval to recurrence and survival among low-risk patients with malignant pleural mesothelioma undergoing surgical macroscopic complete resection. J Thorac Cardiovasc Surg 2013;145:955-63.

67. Rusch VW, Rosenzweig K, Venkatraman E, et al. A phase II trial of surgical resection and adjuvant high-dose hemithoracic radiation for malignant pleural mesothelioma. J Thorac Cardiovasc Surg 2001;122:788-95.

68. Van Schil PE, Opitz I, Weder W, et al. Multimodal management of malignant pleural mesothelioma: where are we today? Eur Respir J 2014;44:754-64.

69. Buduhan G, Menon S, Aye R, et al. Trimodality Therapy for Malignant Pleural Mesothelioma. Ann Thorac Surg 2009;88:870-5.

70. de Perrot M, Feld R, Cho BC, et al. Trimodality therapy with induction chemotherapy followed by extrapleural pneumonectomy and adjuvant high-dose hemithoracic radiation for malignant pleural mesothelioma. J Clin Oncol 2009;27:1413-8.

71. Kapeles M, Gensheimer MF, Mart DA, et al. Trimodality Treatment of Malignant Pleural Mesothelioma: An Institutional Review. Am J Clin Oncol 2018;41:30-5.

72. Thieke C, Nicolay NH, Sterzing F, et al. Long-term results in malignant pleural mesothelioma treated with neoadjuvant chemotherapy, extrapleural pneumonectomy and intensity-modulated radiotherapy. Radiat Oncol 2015;10:267.

73. Mencoboni M, Filiberti RA, Taveggia P, et al. Clinical 
Features and Treatment Outcome of Malignant Pleural Mesothelioma. Oncol Res Treat 2017;40:364-9.

74. Weder W, Stahel RA, Bernhard J, et al. Multicenter trial of neo-adjuvant chemotherapy followed by extrapleural pneumonectomy in malignant pleural mesothelioma. Ann Oncol 2007;18:1196-202.

75. Rea F, Marulli G, Bortolotti L, et al. Induction chemotherapy, extrapleural pneumonectomy (EPP) and adjuvant hemi-thoracic radiation in malignant pleural mesothelioma (MPM): Feasibility and results. Lung Cancer 2007;57:89-95.

76. Krug LM, Pass HI, Rusch VW, et al. Multicenter phase II trial of neoadjuvant pemetrexed plus cisplatin followed by extrapleural pneumonectomy and radiation for malignant pleural mesothelioma. J Clin Oncol 2009;27:3007-13.

77. Van Schil PE, Baas P, Gaafar R, et al. Trimodality therapy for malignant pleural mesothelioma: results from an EORTC phase II multicentre trial. Eur Respir J 2010;36:1362-9.

78. Gomez DR, Rimner A, Simone CB 2nd, et al. The Use of Radiation Therapy for the Treatment of Malignant Pleural Mesothelioma: Expert Opinion from the National Cancer Institute Thoracic Malignancy Steering Committee, International Association for the Study of Lung Cancer, and Mesothelioma Applied Research Foundation. J Thorac Oncol 2019;14:1172-83.

79. Rice DC, Stevens CW, Correa AM, et al. Outcomes after extrapleural pneumonectomy and intensity-modulated radiation therapy for malignant pleural mesothelioma. Ann Thorac Surg 2007;84:1685-92; discussion 1692-3.

80. Ahamad A, Stevens CW, Smythe WR, et al. Intensitymodulated radiation therapy: a novel approach to the management of malignant pleural mesothelioma. Int J Radiat Oncol Biol Phys 2003;55:768-75.

81. Taylor A, Powell M. Intensity-modulated radiotherapywhat is it? Cancer Imaging 2004;4:68.

82. Gomez DR, Hong DS, Allen PK, et al. Patterns of Failure, Toxicity, and Survival after Extrapleural Pneumonectomy and Hemithoracic Intensity-Modulated Radiation Therapy for Malignant Pleural Mesothelioma. J Thorac Oncol 2013;8:238-45.

83. Federico R, Adolfo F, Giuseppe M, et al. Phase II trial of neoadjuvant pemetrexed plus cisplatin followed by surgery and radiation in the treatment of pleural mesothelioma. BMC Cancer 2013;13:22.

84. Stahel RA, Riesterer O, Xyrafas A, et al. Neoadjuvant chemotherapy and extrapleural pneumonectomy of malignant pleural mesothelioma with or without hemithoracic radiotherapy (SAKK 17/04): a randomised, international, multicentre phase 2 trial. Lancet Oncol 2015;16:1651-8.

85. Cho BC, Feld R, Leighl N, et al. A feasibility study evaluating Surgery for Mesothelioma After Radiation Therapy: the "SMART" approach for resectable malignant pleural mesothelioma. J Thorac Oncol 2014;9:397-402.

86. Minatel E, Trovo M, Polesel J, et al. Radical pleurectomy/ decortication followed by high dose of radiation therapy for malignant pleural mesothelioma. Final results with long-term follow-up. Lung Cancer 2014;83:78-82.

87. Rimner A, Zauderer MG, Gomez DR, et al. Phase II study of hemithoracic intensity-modulated pleural radiation therapy (IMPRINT) as part of lung-sparing multimodality therapy in patients with malignant pleural mesothelioma. J Clin Oncol 2016;34:2761.

88. Shaikh F, Zauderer MG, von Reibnitz D, et al. Improved Outcomes with Modern Lung-Sparing Trimodality Therapy in Patients with Malignant Pleural Mesothelioma. J Thorac Oncol 2017;12:993-1000.

89. Nelson DB, Rice DC, Mitchell KG, et al. Return to intended oncologic treatment after surgery for malignant pleural mesothelioma. J Thorac Cardiovasc Surg 2019;158:924-9.

90. Espinoza-Mercado F, Borgella JD, Berz D, et al. Disparities in Compliance With National Guidelines for the Treatment of Malignant Pleural Mesothelioma. Ann Thorac Surg 2019;108:889-96.

91. Nelson DB, Rice DC, Niu J, et al. Predictors of trimodality therapy and trends in therapy for malignant pleural mesothelioma. Eur J Cardiothorac Surg 2018;53:960-6.

92. De Bondt C, Psallidas I, Van Schil PE, et al. Combined modality treatment in mesothelioma: a systemic literature review with treatment recommendations. Transl Lung Cancer Res 2018;7:562-73.

93. Marulli G, Faccioli E, Bellini A, et al. Induction chemotherapy vs post-operative adjuvant therapy for malignant pleural mesothelioma. Expert Rev Respir Med 2017;11:649-60.

94. ClinicalTrials.gov. Pleurectomy/Decortication (Neo) Adjuvant Chemothreapy and Intensity Modulated Radiation Therapy to the Pleura in Patients with Locally Advanced Pleural Mesothelioma: Memorial Sloan Kettering Cancer Center; 2008 [updated August 21, 2020]. Available online: https://clinicaltrials.gov/ct2/show/ NCT00715611

95. Ahamad A, Stevens CW, Smythe WR, et al. Promising 
early local control of malignant pleural mesothelioma following postoperative intensity modulated radiotherapy (IMRT) to the chest. Cancer J 2003;9:476-84.

96. Vogelzang NJ, Rusthoven JJ, Symanowski J, et al. Phase III study of pemetrexed in combination with cisplatin versus cisplatin alone in patients with malignant pleural mesothelioma. J Clin Oncol 2003;21:2636-44.

97. Sandin LC, Eriksson F, Ellmark P, et al. Local CTLA4 blockade effectively restrains experimental pancreatic adenocarcinoma growth in vivo. Oncoimmunology 2014;3:e27614.

98. Fransen MF, van der Sluis TC, Ossendorp F, et al. Controlled local delivery of CTLA-4 blocking antibody induces CD8+ T-cell-dependent tumor eradication and decreases risk of toxic side effects. Clin Cancer Res 2013;19:5381-9.

99. Cantini L, Hassan R, Sterman DH, et al. Emerging Treatments for Malignant Pleural Mesothelioma: Where Are We Heading? Front Oncol 2020;10:343.

100. Cedrés S, Ponce-Aix S, Pardo-Aranda N, et al. Analysis of expression of PTEN/PI3K pathway and programmed cell death ligand 1 (PD-L1) in malignant pleural mesothelioma (MPM). Lung Cancer 2016;96:1-6.

101. Mansfield AS, Roden AC, Peikert T, Sheinin YM, Harrington SM, Krco CJ, et al. B7-H1 expression in malignant pleural mesothelioma is associated with sarcomatoid histology and poor prognosis. J Thorac Oncol 2014;9:1036-40.

102.Popat S, Curioni-Fontecedro A, Polydoropoulou V, et al. A multicentre randomized phase III trial comparing pembrolizumab $(\mathrm{P})$ vs single agent chemotherapy (CT) for advanced pre-treated malignant pleural mesothelioma (MPM): Results from the European Thoracic Oncology Platform (ETOP 9-15) PROMISE-meso trial. Ann Oncol 2019;30:v931.

103. Scherpereel A, Mazieres J, Greillier L, et al. Nivolumab or nivolumab plus ipilimumab in patients with relapsed malignant pleural mesothelioma (IFCT-1501 MAPS2): a multicentre, open-label, randomised, non-comparative, phase 2 trial. Lancet Oncol 2019;20:239-53.

104. Alley EW, Lopez J, Santoro A, et al. Clinical safety and activity of pembrolizumab in patients with malignant pleural mesothelioma (KEYNOTE-028): preliminary results from a non-randomised, open-label, phase $1 \mathrm{~b}$ trial. Lancet Oncol 2017;18:623-30.

105.Disselhorst MJ, Quispel-Janssen J, Lalezari F, et al. Ipilimumab and nivolumab in the treatment of recurrent malignant pleural mesothelioma (INITIATE): results of a prospective, single-arm, phase 2 trial. Lancet Respir Med 2019;7:260-70.

106. Maio M, Scherpereel A, Calabro L, et al. Tremelimumab as second-line or third-line treatment in relapsed malignant mesothelioma (DETERMINE): a multicentre, international, randomised, double-blind, placebocontrolled phase 2b trial. Lancet Oncol 2017;18:1261-73.

107. Calabrò L, Morra A, Giannarelli D, et al. Tremelimumab combined with durvalumab in patients with mesothelioma (NIBIT-MESO-1): an open-label, non-randomised, phase 2 study. Lancet Respir Med 2018;6:451-60.

108. Parra HS, Tixi L, Latteri F, et al. Combined regimen of cisplatin, doxorubicin, and alpha-2b interferon in the treatment of advanced malignant pleural mesothelioma: a Phase II multicenter trial of the Italian Group on Rare Tumors (GITR) and the Italian Lung Cancer Task Force (FONICAP). Cancer 2001;92:650-6.

109. Halme M, Knuuttila A, Vehmas T, et al. High-dose methotrexate in combination with interferons in the treatment of malignant pleural mesothelioma. Br J Cancer 1999;80:1781-5.

110. Bretti S, Berruti A, Dogliotti L, et al. Combined epirubicin and interleukin-2 regimen in the treatment of malignant mesothelioma: a multicenter phase II study of the Italian Group on Rare Tumors. Tumori 1998;84:558-61.

111.Pastan I, Hassan R. Discovery of mesothelin and exploiting it as a target for immunotherapy. Cancer Res 2014;74:2907-12.

112. Ho M, Onda M, Wang QC, et al. Mesothelin is shed from tumor cells. Cancer Epidemiol Biomarkers Prev 2006;15:1751.

113. Hassan R, Remaley AT, Sampson ML, et al. Detection and quantitation of serum mesothelin, a tumor marker for patients with mesothelioma and ovarian cancer. Clin Cancer Res 2006;12:447-53.

114. Robinson BW, Creaney J, Lake R, et al. Mesothelinfamily proteins and diagnosis of mesothelioma. Lancet 2003;362:1612-6.

115.Adusumilli PS, Zauderer MG, Rusch VW, et al. Regional delivery of mesothelin-targeted CAR T cells for pleural cancers: Safety and preliminary efficacy in combination with anti-PD-1 agent. J Clin Oncol 2019;37:2511.

116. Chong EA, Svoboda J, Dwivedy Nasta S, et al. Sequential Anti-CD19 Directed Chimeric Antigen Receptor Modified T-Cell Therapy (CART19) and PD-1 Blockade with Pembrolizumab in Patients with Relapsed or Refractory B-Cell Non-Hodgkin Lymphomas. Blood 2018;132:4198. 117. Mundlos S, Pelletier J, Darveau A, et al. Nuclear 
localization of the protein encoded by the Wilms' tumor gene WT1 in embryonic and adult tissues. Development 1993;119:1329-41.

118.Zauderer MG, Tsao AS, Dao T, et al. A Randomized Phase II Trial of Adjuvant Galinpepimut-S, WT-1 Analogue Peptide Vaccine, After Multimodality Therapy for Patients with Malignant Pleural Mesothelioma. Clin Cancer Res 2017;23:7483-9.

119. Gabrilovich DI, Ciernik IF, Carbone DP. Dendritic cells in antitumor immune responses. I. Defective antigen presentation in tumor-bearing hosts. Cell Immunol 1996;170:101-10.

120.Enk AH, Jonuleit H, Saloga J, et al. Dendritic cells as mediators of tumor-induced tolerance in metastatic melanoma. Int J Cancer 1997;73:309-16.

121.Hegmans JP, Veltman JD, Lambers ME, et al. Consolidative dendritic cell-based immunotherapy elicits cytotoxicity against malignant mesothelioma. Am J Respir Crit Care Med 2010;181:1383-90.

122. Cornelissen R, Hegmans JP, Maat AP, et al. Extended Tumor Control after Dendritic Cell Vaccination with Low-Dose Cyclophosphamide as Adjuvant Treatment in Patients with Malignant Pleural Mesothelioma. Am J Respir Crit Care Med 2016;193:1023-31.

123. Belderbos RA, Baas P, Berardi R, et al. A multicenter, randomized, phase II/III study of dendritic cells loaded with allogeneic tumor cell lysate (MesoPher) in subjects with mesothelioma as maintenance therapy after chemotherapy: DENdritic cell Immunotherapy for Mesothelioma (DENIM) trial. Transl Lung Cancer Res 2019;8:280-5.

124. Seymour LW, Fisher KD. Oncolytic viruses: finally delivering. Br J Cancer 2016;114:357-61.

125.Vachani A, Moon E, Wakeam E, et al. Gene therapy for mesothelioma and lung cancer. Am J Respir Cell Mol Biol 2010;42:385-93.

126. Sterman DH. Gene therapy for malignant pleural mesothelioma. Hematol Oncol Clin North Am 2005;19:1147-73, viii.

127. Pease DF, Kratzke RA. Oncolytic Viral Therapy for Mesothelioma. Front Oncol 2017;7:179.

128. Elshami AA, Kucharczuk JC, Zhang HB, et al. Treatment of pleural mesothelioma in an immunocompetent rat model utilizing adenoviral transfer of the herpes simplex virus thymidine kinase gene. Hum Gene Ther 1996;7:141-8.

129. Sterman DH, Treat J, Litzky LA, et al. Adenovirus- mediated herpes simplex virus thymidine kinase/ganciclovir gene therapy in patients with localized malignancy: results of a phase I clinical trial in malignant mesothelioma. Hum Gene Ther 1998;9:1083-92.

130.Sterman DH, Molnar-Kimber K, Iyengar T, et al. A pilot study of systemic corticosteroid administration in conjunction with intrapleural adenoviral vector administration in patients with malignant pleural mesothelioma. Cancer Gene Ther 2000;7:1511-8.

131. Sterman DH, Recio A, Vachani A, et al. Long-term follow-up of patients with malignant pleural mesothelioma receiving high-dose adenovirus herpes simplex thymidine kinase/ganciclovir suicide gene therapy. Clin Cancer Res 2005;11:7444-53.

132. Muggerud AA, Hallett M, Johnsen H, et al. Molecular diversity in ductal carcinoma in situ (DCIS) and early invasive breast cancer. Mol Oncol 2010;4:357-68.

133. Grosso F, Steele N, Novello S, et al. Nintedanib Plus Pemetrexed/Cisplatin in Patients With Malignant Pleural Mesothelioma: Phase II Results From the Randomized, Placebo-Controlled LUME-Meso Trial. J Clin Oncol 2017;35:3591-600.

134. Scagliotti GV, Gaafar R, Nowak AK, et al. Nintedanib in combination with pemetrexed and cisplatin for chemotherapy-naive patients with advanced malignant pleural mesothelioma (LUME-Meso): a double-blind, randomised, placebo-controlled phase 3 trial. Lancet Respir Med 2019;7:569-80.

135. Kelly WK, O'Connor OA, Krug LM, et al. Phase I study of an oral histone deacetylase inhibitor, suberoylanilide hydroxamic acid, in patients with advanced cancer. J Clin Oncol 2005;23:3923-31.

136. Krug LM, Kindler HL, Calvert H, et al. Vorinostat in patients with advanced malignant pleural mesothelioma who have progressed on previous chemotherapy (VANTAGE-014): a phase 3, double-blind, randomised, placebo-controlled trial. Lancet Oncol 2015;16:447-56.

doi: $10.21037 /$ ccts-20-112

Cite this article as: Di Lena É, Aboalsaud A, Sirois C, Mulder D, Spicer J, Ferri L, Cools-Lartigue J. A narrative review of current treatment strategies and emerging therapies in malignant pleural mesothelioma. Curr Chall Thorac Surg 2021;3:8. 